\title{
Didactiques : les modèles en questions
}

\section{Yves Reuter}

\section{(2) OpenEdition}

Journals

Édition électronique

URL : http://journals.openedition.org/trema/3529

DOI : 10.4000/trema.3529

ISSN : 2107-0997

\section{Éditeur}

Faculté d'Éducation de l'université de Montpellier

\section{Édition imprimée}

Date de publication : 1 octobre 2016

Pagination : 107-112

ISBN : 979-10-96627-01-1

ISSN : 1167-315X

\section{Référence électronique}

Yves Reuter, «Didactiques : les modèles en questions », Tréma [En ligne], 45 | 2016, mis en ligne le 01 janvier 2017, consulté le 30 avril 2019. URL : http://journals.openedition.org/trema/3529 ; DOI : 10.4000/trema.3529

Ce document a été généré automatiquement le 30 avril 2019

Trema 


\title{
Didactiques : les modèles en questions
}

\author{
Yves Reuter
}

1 Il convient de saluer la témérité de Rosine Galluzzo-Dafflon pour avoir initié une journée ${ }^{1}$ sur un objet tel que les modèles, tant cet objet s'avère complexe et subit des traitements variés dans les différentes didactiques. Si cette journée a été particulièrement riche ainsi qu'en atteste ce volume, les contributions qui en sont issues ne sont pas faciles à synthétiser. Deux raisons principales expliquent cela, au moins en partie :

- la maitrise inégale que chacun d'entre nous - moi le premier - peut avoir des différentes didactiques, des différentes disciplines scolaires et de leurs multiples disciplines de référence, ce qui constitue d'ailleurs un problème récurrent dès qu'on s'aventure sur le terrain de la didactique comparée ;

- la diversité des domaines étudiés dans ce numéro, non seulement pour ce qui concerne les didactiques mais aussi pour ce qui concerne les étapes du cursus scolaire : de la maternelle à l'université...

2 Aussi le lecteur me pardonnera, du moins je l'espère, le caractère quelque peu morcelé de cet article « réactif $~^{2}$, sous forme de remarques autour de quelques points: le statut de cette réflexion sur les modèles; les disciplines présentes ou absentes; une tentative de bilan des apports de cette journée et de ce numéro de Tréma; les questions posées et les débats ouverts.

\section{Deux remarques sur le statut de cette réflexion}

Il me semble important de souligner, en premier lieu, que l'émergence de telles journées constitue sans doute une marque de la possible maturité des didactiques ${ }^{3}$, d'une réflexivité qui se construit et se met en débat, avec des analyses fines sur ce que signifie « faire » de la science ou expliquer pour telle discipline de recherche à tel moment de son histoire. De ce point de vue, j'avancerais volontiers que cela participe d'un mouvement qui a vu se multiplier dans les champs des didactiques, et cela depuis quelques années 
déjà, dictionnaires spécialisés, ouvrages consacrés aux méthodes, livres portant sur l'histoire des didactiques, «Que sais- je ? »... Il reste cependant à expliquer le peu de références bibliographiques aux articles et ouvrages didactiques eux-mêmes dans plusieurs des contributions ici présentes, comme si la réflexion sur la scientificité des didactiques ne pouvait se légitimer que de l'extérieur, que d'autres disciplines de recherche...

4 Je m'interrogerai encore sur les "disciplines" présentes dans ce numéro: une contribution en Sciences (Orange-Ravachol) ; trois contributions en Français (Dufays sur la lecture littéraire; Galluzzo-Dafflon sur l'écriture ; Pulido sur l'oral en maternelle); deux sur des "quasi-disciplines» (Frisch en information-documentation, discipline "émergente»; Chabanne en histoire des arts, cette "transdiscipline») ${ }^{4}$. À cela, j'ajouterai deux contributions que j'appellerai, faute de mieux, "méta » : celle de Fabre qui parcourt des territoires philosophico-épistémologiques et celle de Sensevy qui met à l'œuvre certains cadres possibles de la didactique comparée... Cela peut légitimement susciter des interrogations quant à ces présences (entre disciplines «établies» qui manient des modèles depuis bien longtemps et disciplines en questionnement sur leur statut). Cela peut tout aussi légitimement susciter des questions quant aux absences de certaines disciplines ou "sous-disciplines", pourtant pourvoyeuses de modèles depuis bien longtemps (mathématiques, lecture, orthographe, grammaire...). Cela impose en tout cas de s'interroger sur les formes d'organisation des contenus au sein de l'appareil scolaire, entre disciplines, sous-disciplines et quasi-disciplines et, par voie de conséquence, sur les relations entre les didactiques et ces formes d'organisation.

\section{Des apports indéniables}

5 Je souhaite maintenant, sans vouloir ni pouvoir être exhaustif, souligner certains apports indéniables de cette journée.

\section{1. De l'importance des modèles}

6 Ces apports portent en premier lieu sur l'importance des modèles notamment au regard de leurs multiples fonctions qui accompagnent et incarnent les processus de théorisation. Ainsi, les auteurs des diverses contributions présentes dans ce volume ont pu montrer comment les modèles étayent la démarche de compréhension, comment ils peuvent illustrer ou constituer par eux-mêmes des modalités descriptives, comment ils peuvent aider à expliquer ou encore à enseigner (voire à apprendre ?). Ils peuvent aussi orienter l'action (voir, par exemple, les modèles « étapistes », cf. Frisch) ou permettre d'articuler la construction de normes et le repérage des variations... Il resterait, peut-être, à expliquer un peu plus avant les relations entre théories et modèles, par exemple dans ce qui fait qu'une théorie se nourrit ou non de modèles, ou qu'un modèle a un statut théorique ou non...

\section{2. Les modèles comme constructions}

7 Je noterai, comme autre apport significatif, le consensus sur le fait qu'un modèle est toujours une construction qui, en tant que telle, manifeste et induit un point de vue, une perspective (voir la mise au point très éclairante de Fabre sur les modèles - topique, 
dynamique et économique - chez Freud). De fait, le modèle vaut non seulement pour ce qu'il éclaire mais aussi par sa manière d'éclairer. Ainsi que l'écrivait Jean-François Halté (2001, p.15) :

Les divers modèles circulant sur la question [la didactique du français] valent tous pour ce qu'ils donnent à voir. C'est là, à mes yeux, leur principale vertu. Ils éclairent la complexité en mettant au jour telle ou telle dimension: ce sont des points de vue, comme dans le guide Michelin, des points panoramiques à partir desquels on voit... ce que l'on peut voir de ces points. Des manières de voir, toutes réductrices, toutes intéressantes. Les modèles aident à poser de " bonnes " questions ou à formuler de " bons " problèmes.

8 Ici encore, il resterait peut-être à préciser pourquoi et en quoi tous les modèles ne se valent pas...

\section{3. De la diversité des modèles}

9 À la multiplicité des fonctions évoquées précédemment répondent d'autres principes de variation. Ainsi les modèles peuvent porter sur divers objets : tel contenu, telle discipline, voire les didactiques elles-mêmes. Ils peuvent aussi s'actualiser selon des modalités différentes : symboliques ou formelles, réduites ou expansées, simplifiées ou complexes... Leurs modes de présence varient tout autant (Orange-Ravachol, Chabanne) : dans les Instructions officielles, dans les manuels, dans les pratiques d'enseignement, dans les disciplines « de référence »... Cela renvoie, entre autres, à la diversité des destinataires : chercheurs, enseignants, élèves... Tout cela n'est pas sans éclairer des différences (ne serait-ce qu'en fonction des technologies disponibles dans les laboratoires ou dans les classes, par exemple), voire des conflits entre les modèles proposés selon les espaces...

Il resterait, ici encore, à interroger le statut des auteurs de ces modèles : chercheurs, prescripteurs, auteurs de manuels... en constatant, dans cet ouvrage, l'absence des élèves comme constructeurs de modèles...

\section{4. Tensions et risques de dérives}

11 Les apports ont encore résidé dans les tensions qui traversent les modèles et leur construction. Plusieurs d'entre elles ont été particulièrement bien mises au jour entre outil d'investigation pour soi et outil d'explicitation pour d'autres; entre concret et abstrait ; entre spatialité et temporalité (Orange-Ravachol ; Fabre) ; entre morcèlement et intégration ; entre connaissance et action ; entre complexité et lisibilité ; entre « montée vers une complexité heuristique » et "descente simplificatrice» (Orange-Ravachol); entre fonctions (Galluzzo-Dafflon) : scolaire (au regard de la construction de savoirs) et sociale (au regard de la généricité et des fonctions associées); entre usages descriptifs ou prescriptifs (Dufays, Frisch); entre dimensions (Pulido). Ces tensions peuvent d'ailleurs parfois générer des modèles différents chez le même auteur (voir les analyses de Fabre sur les modèles chrono-logique et expressif chez Dewey) ou plusieurs modèles de référence pour la construction d'un unique modèle dans tel ou tel domaine de l'enseignement, par exemple la «pédagogie du langage oral» (Pulido). Tout cela ne manque pas d'entrainer des risques de dérives qui accompagnent structurellement ces modèles, par exemple une dérive analogique ou encore des risques d'imprécision contraires aux visées poursuivies (par exemple, l'imprécision des éléments composant le modèle ou l'imprécision des relations entre ces éléments ${ }^{5}$. 


\section{Questions et débats}

12 Il me reste à revenir, et c'est sans doute là que réside une grande partie de la richesse des journées et des publications de recherche, sur les questions engendrées et les débats générés par ces contributions.

\section{1. Des définitions}

13 La première de ces questions est peut-être celle des définitions. Il me semble en effet que la notion même de modèle est particulièrement difficile à définir, ainsi qu'en témoignent les éléments de synthèse proposés dans Lecourt (1999) ou Serres et Ferrouki (1997). Conséquemment, au fil des chapitres, des définitions différentes, explicites ou implicites, se font jour, oscillant entre «simples» listes et organisations complexes (Chabanne, Orange-Ravachol). Cela pose la question de savoir à quel moment commencent les modèles ou, sous une autre forme, ce qu'on appelle modèle. J'avais ainsi proposé précédemment (Reuter, 2002-2003, p.22), à propos des modélisations en didactique de l'écriture, de retenir les traits principaux suivants : l'objectivation (avec la mise au jour de ce qui n'est pas directement visible dans l'objet ), la mise en articulation des données empiriques et des cadres théoriques, l'organisation (avec la distinction et la mise en relation des composantes), la réduction (via le changement d'échelle et la sélection des traits pertinents), la possibilité de figuration... Cela pose encore la question des relations entre ce qu'on ${ }^{6}$ appelle modèle(s) et les ordres de l'écrit ou de l'oral ou le langage luimême (Sensevy).

\section{2. Des limites des modèles}

14 La seconde question, fondamentale à mes yeux, est appelée par l'idée même de construction et de point de vue évoquée précédemment. Quelles sont les limites des modèles? Que sont-ils obligés de laisser dans l'ombre, rançon de ce qu'ils éclairent ? Et comment les modèles formalisent-ils leur part d'ombre? De cela, il a été fort peu question, comme si la proposition de modèle ne pouvait s'affranchir d'une dimension apologétique. Pourtant, il me semble que la valeur d'un modèle réside tout autant dans l'explicitation de ce qu'il donne à voir et selon quelles modalités que dans l'explicitation de ce qu'il ne donne pas à voir et pour quelles raisons...

\section{3. Des modèles à la modélisation}

Les modèles ont été essentiellement étudiés ici en tant que produits et non en tant que productions, c'est-à-dire en tant que processus, en tant qu'activités d'élaboration. Cela ne permet pas véritablement de penser les opérations et les pratiques de modélisation. Existe-t-il des étapes ou opérations plus ou moins contraintes? Modélise-t-on de la même manière selon les acteurs, les lieux sociaux, les finalités ? Toutes les dimensions sont-elles également « intégrables » (voir Galluzzo-Dafflon sur les questions de temporalité)?

Et, dans ce cadre, quelles références privilégier et comment procéder, par exemple, pour articuler des éléments parfois, voire souvent, hétérogènes (Pulido) : référents théoriques, pratiques sociales de référence, pratiques des élèves, traditions scolaires... Et cela se pose- 
t-il de la même manière pour les chercheurs, pour les formateurs, pour les prescripteurs, pour les enseignants...?

17 Cette question, même si elle ne la recouvre pas totalement, renvoie en partie à celle des relations entre les modèles des différentes disciplines ou sous-disciplines au sein d'une même matière ou entre les matières. Qu'en font les acteurs? Les chercheurs, les prescripteurs, les formateurs, les enseignants, les élèves ? Ces modèles sont-ils mis en articulation ou simplement juxtaposés sans que leurs relations soient pensées? Avec quels effets? Je pense ici par exemple aux différents modes de catégorisation des éléments langagiers selon les disciplines (français, langues "anciennes", langues " vivantes »...) ou encore aux formalisations, explicites ou implicites selon les disciplines, de conduites langagières telles que la description, le récit, l'explication ou encore l'argumentation...

\section{4. De l'histoire des modèles à leurs conditions de validité}

18 L'histoire des modèles dans une discipline donnée me parait tout aussi importante à approfondir (Dufays, Galluzzo-Dafflon). Outre la mise au jour de leur historicité, de leurs conditions de possibilité et des débats sous-jacents, cela pose une question complémentaire, non négligeable lorsqu'on aborde des questions liées à la scientificité des disciplines. Jusqu'où la persistance des modèles dans tel domaine peut-elle constituer une marque de leur « robustesse »? Jusqu'où la disparition d'un modèle témoigne-t-elle de son « dépassement »?

\section{5. Quelles sont les spécificités des modèles didactiques ?}

Il me semble encore qu'une autre question, fondamentale pour les didactiques, n'a été finalement que peu traitée ici et mériterait donc d'être approfondie, lors de journées ultérieures. Quelles sont les spécificités - si elles existent - des modèles des didactiques par rapport aux modèles des autres disciplines de recherche?

La perspective de l'enseignement et/ou des apprentissages, si on suit Dolz et Schneuwly (Dir., 1998), ou d'autres éléments encore, par exemple la mise en interaction des

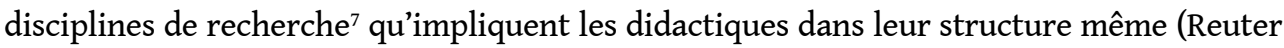
(Dir.), 2007/2013)?

\section{6. Quel cadre pédagogique pour les modèles disciplinaires présentés?}

21 Je ne saurais non plus passer sous silence la question des relations entre disciplines et pédagogie. En effet, ici comme bien souvent, les situations et démarches, implicites ou explicites, présentées par les didacticiens ne renvoient que peu aux pédagogies alternatives (Freinet, de projet...) alors que, par exemple, la référence à Dewey (Fabre) inviterait à penser plus précisément l'articulation possible des disciplines avec ces pédagogies alternatives et, par conséquent, les différences de modélisation que cela est susceptible d'induire. 


\section{Conclusion}

Tout modèle - on l'a vu - existe dans un espace de modèles en conflit(s) et cela quelle que soit la discipline (Orange-Ravachol en didactique de sciences; Dufays pour la lecture littéraire; Chabanne pour l'histoire des arts, entre modèle de la matière et modèle de l'évaluation). Fabre peut ainsi parler du modèle comme d'un «truc à critiquer ». Dès lors, trois remarques s'imposent encore. La première concerne l'importance des fonctions argumentative, apologétique, positionnelle... dans la construction et la défense des modèles. La deuxième porte sur les enjeux, pour son auteur, de l'imposition d'un modèle dans un champ de recherche, ce qui explique, au moins en partie, l'intensité de certaines luttes autour de ces questions. Ma troisième et dernière remarque porte, à la suite de la contribution de Dufays, sur l'importance stratégique de certains modèles pour construire un champ de réflexion. On n'en a pas fini avec les modèles...

\section{BIBLIOGRAPHIE}

Dolz, J. et Schneuwly, B. (Dir.) (1998). Pour un enseignement de l'oral : initiation aux genres formels à l'école. Paris : Esf.

Halté, J. F. (2001). Des modèles de la didactique aux problèmes de la D.F.L.M. In M. MarquilloLarruy (Dir.), Questions d'épistémologie en didactique du Français (langue maternelle, langue seconde, langue étrangère) (pp. 13-19). Poitiers : Université de Poitiers.

Lecourt, D. (Dir.) (1999). Dictionnaire d'histoire et de philosophie des sciences. Paris : PUF.

Marquillo-Larruy, M. (Dir.) (2001). Questions d'épistémologie en didactique du Français (langue maternelle, langue seconde, langue étrangère). Poitiers : Université de Poitiers.

Reuter, Y. (2002-2003). Quelques questions à propos des formalisations de l'écriture en didactique du français, Repères, $n^{\circ}$ 26-27, L'écriture et son apprentissage à l'école primaire, Paris, INRP, 21 - 30.

Reuter, Y. (Dir.) (2007/2013). Dictionnaire des concepts fondamentaux des didactiques. Bruxelles : de Boeck.

Serres M. et Ferrouki N. (Dirs) (1997). Le Trésor. Dictionnaire des Sciences. Paris : Flammarion.

\section{NOTES}

1. Cette journée, qui s'est tenue le 29 janvier 2015 sur le site d'Angers de l'ESPÉ de l'académie de Nantes, était intitulée « Modèles et didactiques. Autour du concept de "modèle" ».

2. J'indique entre parenthèses le nom de l'auteur auquel réfèrent mes remarques.

3. Voir aussi les journées d'étude de janvier 2000 sur la didactique du français (Marquillo-Larruy, 2001).

4. Dans cette journée d'étude, Jean-Marc Lange, traitant des «éducations à », a pu parler d'un « ailleurs disciplinaire ». 
5. Voir, par exemple, l'usage des flèches, sans que leur sens exact soit précisé.

6. Dans cet énoncé et l'énoncé précédent la question du référent du « on » est cruciale.

7. Mise en interaction des théories des contenus, des enseignements et des apprentissages ; mise en interaction des théories sur chacun de ces pôles, ce qui est nécessaire pour éviter l'écueil de l'applicationnisme.

\section{RÉSUMÉS}

Cet article est une synthèse-réaction aux contributions de ce numéro. Il explore donc, après des remarques sur le statut d'une telle réflexion dans le champ des didactiques, les apports de cet ensemble ainsi que les débats ouverts et les questions soulevées.

This paper may be considered as a synthesis of contributions to this latest issue of the journal. The contributions, the open questions and the raised issues of the set of articles are explored after some comments on the status of such a reflexion in the field of the didactics.

INDEX

Keywords : didactics, models, theorizing.

Mots-clés : didactiques, modèles, théorisation

\section{AUTEUR}

\section{YVES REUTER}

Professeur des universités, Université Charles de Gaulle, Lille 3, Laboratoire Théodile-CIREL EA 4354 